\title{
Construccionismo, conocimiento y realidad: una lectura crítica desde la Psicología Social
}

\author{
Dr. Juan Sandoval Moya
}

Doctor en Psicología Social, Universidad Complutense de Madrid Universidad de Valparaíso

juan.sandoval@uv.cl

Resumen

El presente artículo aborda la perspectiva del construccionismo, desde el punto de vista de la psicología social. Se presentan sus principales argumentos y las críticas que se le han hecho a esta postura.

Abstract

This article discusses the perspective of constructionism, from the point of view of the social psychology. It presents the main arguments and criticisms that have been made to this position.

Palabras Clave: Construccionismo, psicología social, epistemología, conocimiento, críticas

Keywords: Constructionism, social psychology, epistemology, knowledge, critiques

\section{Presentación}

El uso de la metáfora de la construcción para referirse a los fenómenos psicológicos y/o sociales no es ni mucho menos una invención de la psicología social. La pluralidad de usos de la raíz construc- ha sido tan extensa en la filosofía y las ciencias sociales que ya hace algunos años Tomás Ibáñez trato de referirse a ellos como una verdadera "galaxia constructivista". Podríamos decir que esta "galaxia" es tan heterogénea que no es posible encuadrarla en una pura disciplina, paradigma o corriente teórica.

Desde el principio de esta presentación, entonces, declaramos que el construccionismo no puede ser analizado como una teoría en el sentido clásico, es decir, en tanto conjunto articulado de proposiciones sobre un aspecto de la realidad social o psicológica que es posible de analizar con los métodos convencionales de la investigación científica. Por el contrario, el construccionismo más bien responde a un conjunto de perspectivas que no necesariamente constituyen un todo coherente y contrastable. Por ello, cuando utilizamos el término "perspectivas" en plural, lo hacemos con el explicito objetivo de evitar "reificar" al construccionismo como un enfoque uniforme y totalmente estabilizado. Podríamos decir que para comprender la unidad en la diversidad del construccionismo, requerimos más que una mirada homogénea, una "mirada caleidoscópica" (Ema y Sandoval, 2003).

Sin embargo, después de varias décadas de desarrollo en Estados Unidos, Europa y América latina, en la actualidad, el construccionismo puede ser reconocido como un programa complejo con expresiones en varios ámbitos de la psicología y las ciencias sociales. En efecto, como propuesta crítica dentro de la psicología social, el 
construccionismo ha ido sedimentando como un conjunto de perspectivas claramente identificables, de modo que nadie dudaría que está ahí, que puede ser visto; y que puede ser considerado como objeto de análisis crítico de esta presentación.

En este contexto, el objetivo de esta comunicación es analizar críticamente el desarrollo y proyección de las perspectivas construccionistas en Psicología Social. Podríamos decir que el sentido último que moviliza esta reflexión es la realización de la crítica sobre la crítica, es decir, llevar al construccionismo a operar como enfoque crítico de sus propias propuestas teóricas, ya que la desconstrucción de sus propuestas resulta una tarea prioritaria en la formulación de un proyecto propiamente construccionista.

\section{Acerca de la perspectiva construccionista}

En 1973 el artículo de Kenneth Gergen "Social Psychology as History" marcó el inicio de una profunda renovación de la psicología social. En aquel artículo, Gergen contrastaba la Psicología Social con el modelo dominante de las ciencias naturales, afirmando que si bien en estas ciencias era posible el conocimiento acumulativo y la aplicación de las metodologías experimentales, en la Psicología Social, debido a que se propone abordar hechos históricos de alta variabilidad, no resultaba posible la acumulación de conocimientos según los cánones tradicionales del discurso de la ciencia (Sandoval, 2009).

El argumento de Gergen en este famoso artículo es simple: si las sociedades humanas son históricamente cambiantes, también lo debían ser los significados que las personas le atribuyen a la realidad, de modo que, si los significados influyen en las acciones y decisiones de las personas que forman esas sociedades, el propio conocimiento científico, que justamente se caracteriza por dar nuevos sentidos a la realidad, tendría la capacidad de afectar la manera como entendemos nuestro mundo. Gergen llegaba así a la conclusión de que el conocimiento que se obtiene en la psicología social es de carácter histórico.

A partir de este artículo original podemos identificar una línea de trabajo que aborda la crítica y "desconstrucción" del conocimiento psicosociológico tradicional y que el propio autor denomina como construccionismo social ${ }^{1}$. Sin embargo, tal como señala Gergen (1996) en su análisis de la evolución de la teoría psicológica contemporánea, el construccionismo no pretende ser una teoría propiamente tal, sino más bien, un intento metateórico por construir una alternativa a la hegemonía del empirismo en la epistemología, del conductismo y del cognitivismo en la teoría y del experimentalismo en la metodología, la trilogía que fundamenta el núcleo de inteligibilidad de la psicología moderna.

Esta crítica al núcleo de inteligibilidad de la psicología se desarrolla en varios niveles y a partir de la recuperación por parte del construccionismo de diversos desarrollos teóricos propuestos fuera del campo disciplinario de la psicología:

- En primer lugar, como consecuencia de los efectos del giro lingüístico, el construccionismo realiza una crítica radical a la idea que el lenguaje tendría la capacidad para vehiculizar los contenidos mentales o para representar los hechos sociales; 
- En segundo lugar, a partir de la lectura de autores como Ludwig Wittgenstein, el construccionismo hace una crítica profunda a la creencia de que la mente contenga representaciones que realmente reflejan lo que es el mundo externo;

- En tercer lugar, tras los planteamientos de Tomas Kuhn y Imre Lakatos en la filosofía de la ciencia, los enfoques construccionistas se hacen parte de la crítica a la concepción progresiva y acumulativa del pensamiento científico;

- Y en cuarto lugar, a partir de los planteamientos de autores tan diversos como Michel Foucault o Richard Rorty, el construccionismo se pliega a una visión crítica de la noción moderna de la verdad.

A partir de estas perspectivas críticas, la elaboración de un nuevo núcleo de inteligibilidad para la psicología pasaría por la formulación de una verdadera epistemología social, donde la producción, fundamentación y validación del conocimiento quedaría radicada en las vicisitudes del proceso social, expresadas en lo que el propio Gergen propone como un enfoque sobre la posesión comunitaria del conocimiento. Efectivamente, a través de estas proposiciones, el construccionismo se hace heredero al mismo tiempo que toma distancia crítica de los aportes señeros de la Escuela crítica de Frankfurt, del estructuralismo y el post-estructuralismo, del pragmatismo y de la sociología del conocimiento, postulando una relación de dependencia entre conocimiento y realidad, a partir de la cual resultaría insostenible la creencia de que el mundo pueda existir con independencia de las condiciones sociales involucradas en su propio conocimiento. De ahí que el construccionismo se autodefina como una verdadera "crítica social":

"es a través de una apreciación crítica del lenguaje como podemos alcanzar la comprensión de nuestras formas de relación con la cultura y, a través de él, abrir un espacio a la consideración de las alternativas futuras. En lugar de considerar la crítica como reveladora de los intereses sesgados podemos considerarla como aclaradora de las consecuencias pragmáticas del propio discurso" (Gergen, 1996: 71)

De este modo, los planteamientos construccionistas se proponen desarrollar una postura teórica y epistemológica vinculada a la crítica y la "desconstrucción", donde el análisis crítico ya no se propone como objetivo levantar el velo de los intereses y los prejuicios que encubren la realidad, sino más bien explorar cómo podrían concebirse de otra manera las cosas y qué tipo de relaciones se podrían establecer a partir de esas nuevas concepciones sobre el mundo y nosotros mismos (Crespo, 2003).

Lo que se propone el construccionismo, entonces, es desarrollar una perspectiva alternativa al enfoque individual del conocimiento, permitiendo analizar el rol que juega el saber compartido por una comunidad en la mantención y reproducción de la realidad. Si el conocimiento radica en la relación que se establece en una comunidad de interpretes: ¿en qué sentido podemos hablar de un conocimiento libre de las marcas sociales e históricas de su producción?, ¿En qué sentido el conocimiento de la psicología -y demás ciencias sociales- es un saber validado por una retórica que se articula en el seno de un núcleo de inteligibilidad en crisis?

Como nos sugiere Tomas Ibáñez (2001), para el construccionismo, el objetivo fundamental ha sido ejecutar un ejercicio crítico que permita echar las bases para el desarrollo de una nueva perspectiva ligada a la metáfora de la construcción. Desde una perspectiva esquemática, podemos decir que esta psicología de la construcción social 
propone una afirmación doble: por un lado, sostiene que la realidad social no tiene necesidad de ser del modo como ha sido o actualmente es, porque es el resultado de la actividad humana; y por otro lado, propone que el conocimiento cotidiano o la representación científica que tenemos de esa realidad social, no es un mero reflejo, sino un elemento constitutivo de lo que la realidad social es.

Más allá de su diversidad, el construccionismo, asumiendo una clara inspiración en las perspectivas posmodernas, define la noción de "acción discursiva" como el objeto de investigación privilegiado para estudiar estos procesos de construcción social, eliminando progresivamente de su repertorio conceptual categorías metafísica y mentalistas para dar cuenta de la subjetividad, y categorías materialistas para dar cuenta de la realidad social.

\section{Rebasando los reduccionismos construccionistas}

Podríamos decir que las perspectivas construccionistas han sido muy agudas a la hora de analizar y desconstruir los sistemas de significados y los repertorios de interpretación con los cuales se instituye la realidad social, desvelando su carácter contingente e histórico. Sin embargo, han presentado dificultades a la hora de explicar el origen y la transformación de las relaciones sociales de carácter más estructural, incurriendo en un verdadero silencio sobre los elementos no lingüísticos de la realidad.

Como plantea Fernando García Selgas (2003), el problema con las propuestas construccionistas surge cuando nos preguntamos por el origen del proceso de construcción social, ya que en la mayoría de los casos, podemos constatar que este proceso es de carácter simbólico, se realiza a partir de algún tipo de sujeto cuyo carácter construido no se explicita totalmente y sus límites parecen ser inexistentes, tal que no habría cosa, humana o no humana, que se le pudiera resistir.

Desde nuestra perspectiva, el construccionismo ha creado una metáfora de la "construcción" que opera con una naturaleza final: el discurso, y un lugar último para su producción: lo social, ubicándola en una suerte de omnipotencia social que omite a otros "agentes" del proceso de construcción. Esta tendencia a una suerte de reduccionismo socio-discursivo, paradójicamente, hace aparecer por acción u omisión del propio construccionismo una brecha irreconciliable y esquiva, una brecha que se transforma en un verdadero foso infranqueable desde todas sus orillas, una brecha que pone por un lado a la subjetividad, al lenguaje y a la mente, y les opone como mundos extraños y contradictorios al objeto, la materialidad y el cuerpo. Como nos señala acertadamente Bruno Latour:

"Pese a los miles de libros que los filósofos del lenguaje han arrojado al abismo que separa el lenguaje del mundo, no se aprecian signos de que la sima pueda colmarse. El misterio de la referencia entre los dos (únicos) ámbitos del lenguaje y del mundo sigue siendo tan oscuro como antes, sólo que ahora tenemos una versión increíblemente sofisticada de lo que sucede en uno de los dos polos -el del lenguaje, la mente, el cerebro y, últimamente, incluso, la sociedad- y una versión totalmente raquítica sobre lo que ocurre en el polo opuesto, esto es, nada" (2001: 178).

A partir de lo anterior, creemos que la única manera de superar esta brecha es trascendiendo cualquier forma de reduccionismo, asumiendo explícitamente que no resulta posible seguir intentando separar las implicaciones constitutivas entre sujeto y objeto, y reconociendo que la única vía realmente novedosa de entender la relación 
entre nuestras prácticas de significación y el mundo, es asumir que tanto nuestras prácticas como el mundo se constituyen mutuamente a partir de una peculiar forma de relación, cuya naturaleza es simbólica y material al mismo tiempo.

Nuestra propuesta sería que debemos centrar el análisis psicosociológico en la construcción "socio-natural" de la realidad, marcando un giro con respecto a las tradiciones objetivista, pero también subjetivistas de la psicología social. Esta perspectiva que en otra parte hemos denominada como "acción situada" (Sandoval, 2004), implicaría una doble proposición con respecto al conocimiento y el mundo; a saber:

En primer lugar, que un hecho es construido por medio de prácticas reales de significación, y que por lo tanto, como todo acto de construcción, requiere de "materiales" que se articulan a partir de un trasfondo sedimentado en nuestros cuerpos y en nuestra forma de vida. Para analizar esta noción de trasfondo, podemos poner en confluencia dos niveles de expresión fundamental: por un lado, un proceso de configuración de un saber práctico, que tiene que ver con un modus operandis subjetivo y corporal que posibilita la acción, y por otro, un proceso de estructuración de reglas y relaciones de poder que definen posiciones y condiciones de posibilidad para la acción.

En segundo lugar, que la relación entre el conocimiento y el mundo no se constituye a través de una representación mental ni una construcción lingüística, sino por medio de un proceso de "articulación". Es decir, el mundo no se aprehende ni se construye, en el sentido literal, sino que es algo con lo cual dialogamos, negociamos y nos mezclamos a través del conocimiento, porque ambos, conocimiento y mundo, son ámbitos incompletos e interdependientes que se constituyen en tanto tales, en el momento mismo de la articulación.

A partir de ambas proposiciones se estructura la perspectiva de la acción situada, la cual propone que el proceso de construcción social debe ser entendido como la "articulación" de unos elementos simbólicos y materiales dispersos en un "trasfondo", elementos que por definición no contarían con una identidad definida a priori, sino que ésta sería el resultado de la propia "articulación" que se produce en el momento de la acción (Sandoval, 2004).

\section{Comentarios finales}

Al finalizar nuestro análisis, podemos concluir que la revolución teórica representada por el construccionismo en la psicología social, estableció las condiciones de posibilidad para preguntarnos desde y sobre el saber psicosocial de una manera que hasta su formulación resultaba insospechada. Categorías como discurso, subjetividad e historicidad aparecen cada vez con más frecuencia en los estudios de la nueva psicología social construccionista, representando una verdadera actualización disciplinaria de este campo del saber con respecto a los debates de la filosofía y demás ciencias sociales.

Sin embargo, nuestro análisis también ha planteado la necesidad de rebasar las propuestas construccionistas en busca de metáforas que puedan escapar con mayor facilidad de las tendencias dualistas que encontramos escondidas, incluso en las propias perspectivas construccionistas. Este rebasamiento es el que nos permite reafirmar que el sujeto y el mundo, lo social y lo natural, lo humano y lo tecnológico, no constituyen esferas ontológicas totalmente separadas. 
Por el contrario, nuestra propuesta final ha sido que los sujetos estamos situados en un mundo que hacemos y nos hace a la vez, de modo que una práctica social no es reducible ni a la imagen objetivista de que hacemos algo en el mundo, pero tampoco a la metáfora humanista de que "nosotros" -los seres humanos- hacemos totalmente el mundo. Más bien, su comprensión requiere de nuevas metáforas como las insinuadas en esta presentación, porque como señala Casado y Gatti: "(...) la actividad cognitiva no se sitúa ni del lado del objeto ni del lado del sujeto, sino precisamente en la mutua negociación que los constituye y que perfila las fronteras que permiten pensarlos automáticamente" (2001: 154).

Podemos concluir, finalmente, que la perspectiva propuesta en esta presentación implica necesariamente un cambio de mirada en el entendimiento del proceso de construcción social de la realidad. Contra la rigidez de nociones como "estructura", "cognición", "sistema" o "función", este análisis propone seguir a los actores en sus múltiples actividades de "articulación", sobrepasando las fronteras predefinidas de los sistemas y las funciones, e incluso redefiniéndolas desde unas reglas del juego que nunca están dadas de una vez y para siempre. RM

\section{Referencias}

Casado, E. \& Gatti, G. (2001). Viaje por las fronteras del campo sociológico. Una cartografía de la investigación social. Política y Sociedad, 36(1), 151 -171.

Crespo, E. (2003). El construccionismo y la cognición social. Metáforas sobre la mente. Política y Sociedad, 40(1), 15-22.

Ema, J. E. \& Sandoval, J. (2003). Mirada caleidoscópica al construccionismo social. Política y Sociedad, 40(1), 5-14.

García Selgas, F. (2003). Para una ontología política de la fluidez social: el desbordamiento de los constructivismos. Política y Sociedad, 40(1), 27-55.

Gergen, K. (1973). Social psychology as history. Journal of Personality and Social Psychology, 26, 309-320.

Gergen, K. (1996) Realidades y relaciones. Aproximaciones a la construcción social. Barcelona: Paidós. [1994]

Ibáñez, T. (2001) Municiones para disidentes. (Realidad - Verdad - Política). Barcelona: Gedisa.

Latour, B. (2001) La Esperanza de Pandora. Barcelona: Gedisa.

Sandoval, J. (2004) Representación, discursividad y acción situada. Introducción crítica a la psicología social del conocimiento. Valparaíso: Editorial de la Universidad de Valparaiso.

Sandoval, J. (2009) Una lectura a la crisis y Reconstrucción de la Psicología Social. Cuadernos de Postgrado en Psicología UV, 1, 18-29.

(URL: http://www.psicologia-uv.cl/post documentos.php) 


\section{Notas}

1 Kenneth Gergen identifica cinco programas ligados a las propuestas constructivas: el constructivismo radical de Ernst von Glazersnfeld, el constructivismo de Jean Piaget y George Kelly, el constructivismo social de Lev Vygotsky, Jerome Bruner y Serge Moscovici; el construccionismo sociológico de Henri Giroux y Nikolas Rose; y el construccionismo social representado por su propia propuesta teórica (1996: 60; nota 30).

Revista Mad. No 23, Septiembre de 2010. Departamento de Antropología. Universidad de Chile http://www.revistamad.uchile.cl/23/sandoval_04.pdf 